\title{
Selesconol, a Fungal Polyketide That Induces Stem Cell Differentiation
}

Ai Hua Zhang, Rui Tan, Nan Jiang, Kaidiriye Yusupu, Gang Wang, Xin Lei Wang, and Ren Xiang Tan

*Corresponding author. E-mail: $\underline{\text { rxtan@nju.edu.cn }}$

\section{Table of Contents}

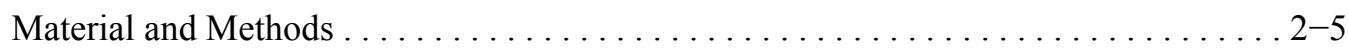

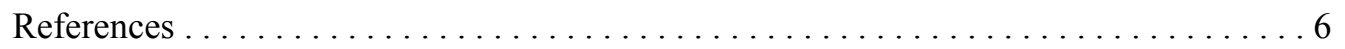

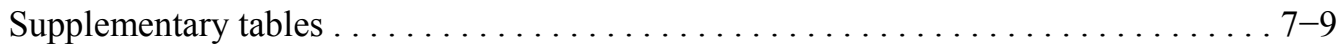

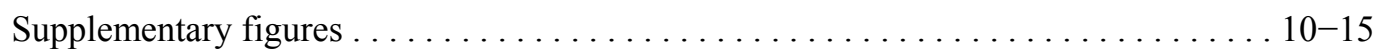




\section{Materials and methods}

\section{General experimental procedures}

As described. ${ }^{1}$

\section{Fungal Strain}

The WT, pksTLKO and lacTLKO strains of Daldinia eschscholzii IFB-TL01 were constructed and preserved as described before. ${ }^{1 \mathrm{c}}$

\section{Cultivation and metabolite isolation}

D. eschscholzii IFB-TL01 was grown on $500 \mathrm{~L}$ malt-extract broth $(20 \mathrm{~g} / \mathrm{L}$ malt extract, $20 \mathrm{~g} / \mathrm{L}$ sucrose, $1 \mathrm{~g} / \mathrm{L}$ peptone) and incubated for 8 days at $25^{\circ} \mathrm{C}$ and $100 \mathrm{rpm}$. The broth was collected, concentrated and extracted with EtOAc to afford a dark brown gum (302.7 g). The broth extract was separated by column chromatography $(\mathrm{CC})$ over silica gel $(1000 \mathrm{~g}, 300-400$ mesh, $50 \times 15 \mathrm{~cm})$ eluted with petroleum ether/acetone mixtures ( $\mathrm{v} / \mathrm{v} 50: 1,20: 1,10: 1,5: 1,2: 1,1: 1,0: 100)$. Further purification of the ' $5: 1$ ' - and ' $10: 1$ '-eluted CC fractions was accomplished by gel filtration over Sephadex LH-20 in $\mathrm{MeOH}$ to afford selesconol $(\mathbf{1}, 65 \mathrm{mg})$ as a red powder. LC-UV-MS screenings were performed to trace the presence of $\mathbf{1}$ in the subfractions.

\section{Single crystal X-ray diffraction}

The structures were solved by direct methods (SHELXS-97) and refined using full-matrix least-squares difference Fourier techniques. Crystallographic data in CIF format have been deposited in the Cambridge Crystallographic Data Centre [available free of charge at http://www.ccdc.cam.ac.uk/deposit or from the CCDC, 12 Union Road, Cambridge CB21EZ, UK; fax: (+44) 1223-336-033; or e-mail: deposit@ccdc.cam.ac.uk].

\section{Crystal data}

Selesconol (1). Diffraction measurements were performed at $296 \mathrm{~K}$ on an Agilent SuperNova diffractometer equipped with $\mathrm{Cu}-K_{\alpha}$ radiation $\left(\lambda=1.54178 \AA\right.$ ). $\mathrm{C}_{29} \mathrm{H}_{18} \mathrm{O}_{6}, M_{\mathrm{r}}=462.43$, triclinic, space group $P-1, a=9.2923$ (7) $\AA, b=11.3936$ (8) $\AA, c=11.6396$ (9) $\AA, V=1042.46$ (15) $\AA^{3}, Z=2, D_{x}=$ $1.473 \mathrm{~g} / \mathrm{cm}^{3}, \mu=0.852 \mathrm{~mm}^{-1}$ and $F(000)=480.0$; crystal dimensions: $0.26 \times 0.18 \times 0.16 \mathrm{~mm}^{3} ; 3069$ 
unique reflections with 3387 obeying the $I \geq 2 \sigma(I) ; R 1=0.040, w R 2=0.112, S=1.056$; supplementary publication no. CCDC-1063723.

\section{Computational details}

ECD calculation. Density functional theory (DFT) at B3LYP/6-31G (d,p) level was employed to optimize the geometries of the studied systems, taking crystal structures as the starting configurations. The solvent effects on the electronic structures of the concerned systems were evaluated by quantum chemistry method through the polarizable continuum model (PCM, dielectric constant $\varepsilon=36.64$ for $\left.\mathrm{CH}_{3} \mathrm{CN}\right){ }^{2}$ Then, the corresponding excited-state calculations were performed at the ground-state optimized geometries. Time-dependent DFT in combination with PCM model (TD-DFT/PCM) with the same basis set was carried out to calculate the spin-allowed excitation energy and rotatory strength of the lowest 100 excited states. The UV and ECD spectra were generated using the program SpecDis ${ }^{3}$ by applying a Gaussian band shape with the width of $0.20 \mathrm{eV}$, from oscillator strengths and dipole-velocity rotational strengths, respectively.

\section{Preparation of intra- and extracellular fungal proteins}

Intracellular protein. The intracellular protein was extracted at $4{ }^{\circ} \mathrm{C}$ from the conidia of the fungus cultivated in ME medium at $28{ }^{\circ} \mathrm{C}$ with orbital shaking at $120 \mathrm{rpm}$ for 4 days. The mycelium was collected by filtering the culture with a Buchner funnel, washed twice with distilled water, frozen in liquid nitrogen, and ground into fine powder, which was suspended in PBS buffer $(\mathrm{pH}=7.0)$, applied to ultra-sonication for $30 \mathrm{~min}$ and then centrifuged at $10000 \mathrm{rpm}$ for $20 \mathrm{~min}$. The supernatant was collected and the protein concentration was assessed using the BCA Protein Assay Kit.

Extracellular protein. The fungus were cultivated for 4 days and filtrated in a Buchner funnel. The filtrate was centrifuged to get rid of the insoluble material, and the supernatant was precipitated with $\left(\mathrm{NH}_{4}\right)_{2} \mathrm{SO}_{4}$. The precipitated protein was collected by centrifugation at $10,000 \mathrm{rpm}$ for $30 \mathrm{~min}$, and the resultant precipitate was dissolved in citric acid-disodium hydrogen phosphate buffer $(\mathrm{pH}=5.0)$, followed by the protein quantification as mentioned above.

\section{Test for the inter-conversion between dalesconol A and selesconol (1)}

Dalesconol A (or selesconol) was dissolved in DMSO at $50 \mathrm{mg} / \mathrm{mL}$, and $20 \mu \mathrm{L}$ of the solution were added to each millilitre of reaction buffer containing $100 \mu \mathrm{L}$ intra- or extra-cellular proteins solution 
and $880 \mu \mathrm{L}$ of PBS buffer. The in vitro transformation was carried out in PBS buffers $(\mathrm{pH}=7.0)$ at $28^{\circ} \mathrm{C}$ for $10 \mathrm{~h}$. The reactions were quenched by adding $10 \mu \mathrm{L} \mathrm{HCl}$ and extracted by equal volume of EtOAc. The organic phases were dried in vacuo and dissolved in $\mathrm{MeOH}$ followed by LC-MS analysis.

\section{Animals and Regents}

Male SD rats (3-6 weeks old,JIANYANG DASHUO), DMEM medium with low glouse (Hyclone); FBS (Gibco); Penicillin-streptomycin (Hyclone); Taq DNA polymerase (TIANGEN); dNTP (Promega); TRIzol® Reagent (Ambion $\left.{ }^{\circledR}\right)$; Chloroform (Sigma); 2-Propanol (Sigma); Absolute alcohol (Sigma); GoldScript cDNA systhesis Kit (Invitrogen); DMSO (Sigma); SsFast ${ }^{\mathrm{TM}}$ Eva Green ${ }^{\circledR}$ Super Mix (BIO-RAD); RA (Retinoic Acid, Sigma).

\section{Isolation and primary culture of mesenchymal stem cells (MSCs)}

To isolate bone marrow-derived rat MSCs, male SD rats (3-6 weeks old) were sacrificed by cervical dislocation and their femurs and tibiae were carefully dissected from the trunk of the body. Then, the femurs and tibiae were immersed in 75\% ethanol for $15 \mathrm{~min}$ and rinsed cleanly with PBS. Muscle and connective tissue were removed from the tibiae and femur and the ends of tibiae and femur were cut. Bone marrow was harvested by inserting a 27-gauge needle into one end of the bone and flushing with $5 \mathrm{~mL}$ low glucose DMEM (Gibco). Cells $\left(25 \times 10^{6}\right.$ cells $\left./ \mathrm{mL}\right)$ were plated into $100-\mathrm{mm}$ culture dishes in DMEM containing 15\% FBS (Gibco), 1\% L-glutamine (Gibco, USA), $100 \mu / \mathrm{mL}$ penicilli and $100 \mu / \mathrm{mL}$ streptomycin. Cultures were incubated at $37{ }^{\circ} \mathrm{C}$ with $5 \% \mathrm{CO}_{2}$ in a humidified chamber. After different culture times, the nonadherent cells were removed by changing medium and replacing with fresh medium. After 2 weeks of initiating culture, the adherent spindle-shaped cells (MSCs) reached $70-80 \%$ confluence and were harvested for further experiments. Only MSC that were subcultured to the third generation were used for the experiments.

\section{Neural cell differentiation of MSC}

To determine whether the bone marrow-derrived MSCs could differentiate into neural cell, the MSCs were culture in a medium containing $(-)-\mathbf{1},(+)-\mathbf{1}$ and RA for 3 days, 5 days and 7 days, wherein RA is a positive control. The medium was replaced once every three days for neural cell differentiation of MSCs. However, no substantial effect could be discerned with dalesconols A and B which were co-assayed identically.

\section{Real-time quantitative PCR (Q-PCR)}

Cells were homogenized in Trizol reagent (Invitrogen Life technologies, USA). Then total RNA was 
treated by DNaseI (Invitrogen Life technologies, USA) and subjected to quantitative PCR, which was performed with the BioRad CFX96 TouchTM Real-Time PCR Detection System (BioRad, CA) using iQTM SYBR ${ }^{\circledR}$ Green Supermix (BioRad, CA), and threshold cycle numbers were obtained using BioRad CFX Manager software. The program for amplification was 1 cycle of $95{ }^{\circ} \mathrm{C}$ for 2 min followed by 40 cycles of $95^{\circ} \mathrm{C}$ for $10 \mathrm{~s}, 60^{\circ} \mathrm{C}$ for $30 \mathrm{~s}$, and $95^{\circ} \mathrm{C}$ for $10 \mathrm{~s}$. The primer sequences used in this study were as follows: Nestin forward AAGGGGATGCCGGGAAGGAAGACTG, reverse CAGAGGCCCCTGACCCCCACCACT; NSE forward GCCCGTGCCGGCCTTTAATGTGATC, reverse ATCGGGAAGGGTCAGCGGGAGACTT; and $\beta$-actin forward CTCCTCCCTGGA GAAGAGCTA, reverse CCTTCTGCATCCTGTCGGCAA.

\section{Immunofluorescence cytochemistry}

After being treated by (+)-1, (-)-1 and RA 3 days, the differentiated rMSCs were fixed in $4 \%$ paraformaldehyde for $30 \mathrm{~min}$ at room temperature, washed three times with PBS for 5 min each and pretreated with $3 \% \mathrm{H}_{2} \mathrm{O}_{2}$ for 10 min. After three 5-min washes with PBS, cells were blocked in PBS containing 3\% goat serum and $0.5 \%$ Triton X-100 for $1 \mathrm{~h}$ at room temperature. Afterwards, cells were incubated with primary antibodies Nestin (1:200, Cy3-labeled antibody, red fluorescence) and NSE (1:200, FITC-labeled antibody, green fluorescence) for $24 \mathrm{~h}$ at $4{ }^{\circ} \mathrm{C}$. The coverslips were counterstained with DAPI (Nuclear dye, blue fluorescence) and imaged with a confocal laser scanning microscope (Olympus, Lake Success, NY). 


\section{Reference:}

(1) (a) Zhang, Y. L.; Ge, H. M.; Zhao, W.; Dong, H.; Xu, Q.; Li, S. H.; Li, J.; Zhang, J.; Song, Y. C.; Tan, R. X. Angew. Chem. Int. Ed. 2008, 47, 5823-5826. (b) Zhang, Y. L.; Zhang, J.; Jiang, N.; Lu, Y. H.; Wang, L.; Xu, S. H.; Wang, W.; Zhang, G. F.; Xu, Q.; Ge, H. M.; Ma, J.; Song, Y. C.; Tan, R. X. J. Am. Chem. Soc. 2011, 133, 5931-5940. (c) Fang, W.; Ji, S.; Jiang, N.; Wang, W.; Zhao, G. Y.; Zhang, S.; Ge, H. M.; Xu, Q.; Zhang, A. H.; Zhang, Y. L.; Song, Y. C.; Zhang, J.; Tan, R. X. Nat. Commun. 2012, 3, 1039.

(2) (a) Cossi, M.; Barone, V.; Cammi, R.; Tomasi, J. J. Chem. Phys. Lett. 1996, 255, 327-335. (b) Cossi, M.; Barone, V. J. Chem. Phys. 1998, 109, 6246-6254. (c) Cossi, M.; Rega, N.; Scalmani, G.; Barone, V. J. Chem. Phys. 2001, 114, 691-5701. (d) Mennucci, B.; Cances, E.; Tomasi, J. J. Chem. Phys. B 1997, 101, 10506-10517. (e) Mennucci, B.; Cammi, R.; Tomasi, J. J. Chem. Phys. 1999, 110, 6858-6870. (f) Cances, E.; Mennucci, B.; Tomasi, J. J Chem Phys. 1997, 107, 3032-3041. (g) Cammi, R.; Mennucci, B. J. Chem. Phys. 1999, 110, 9877-9886.

(3) Bruhn, T.; Hemberger, Y.; Schaumlöffel, A.; Bringmann, G. SpecDis version 1.50, 2010, University of Wuerzburg: Germany. 
Table S1. ${ }^{1} \mathrm{H}$ - and ${ }^{13} \mathrm{C}-\mathrm{NMR}$, and $\mathrm{HMBC}$ data for selesconol (1) in $\mathrm{CDCl}_{3}$.

\begin{tabular}{|c|c|c|c|}
\hline position & $\delta_{\mathrm{H}}$ (multiplicity, $J$ in $\mathrm{Hz}$ ) & $\delta_{\mathrm{C}}$ & HMBC \\
\hline 1 & & 205.4 & \\
\hline 2 & & 120.6 & \\
\hline 3 & & 162.6 & \\
\hline 4 & $7.20(\mathrm{~d}, 8.5)$ & 121.3 & $\mathrm{C} 2, \mathrm{C} 6$ \\
\hline 5 & $7.41(\mathrm{t}, 8.5)$ & 135.7 & $\mathrm{C} 3, \mathrm{C} 7$ \\
\hline 6 & $6.38(\mathrm{~d}, 8.5)$ & 125.9 & $\mathrm{C} 2, \mathrm{C} 4, \mathrm{C} 8$ \\
\hline 7 & & 137.7 & \\
\hline 8 & & 149.0 & \\
\hline 9 & & 133.2 & \\
\hline 10 & $7.14(\mathrm{~d}, 10.1)$ & 143.4 & $\mathrm{C} 8, \mathrm{C} 12, \mathrm{C} 18$ \\
\hline 11 & $6.42(\mathrm{~d}, 10.1)$ & 125.7 & $\mathrm{C} 9, \mathrm{C} 13$ \\
\hline 12 & & 189.1 & \\
\hline 13 & & 115.0 & \\
\hline 14 & & 163.8 & \\
\hline 15 & $7.28(\mathrm{~d}, 8.9)$ & 118.6 & $\mathrm{C} 13, \mathrm{C} 17$ \\
\hline 16 & $7.96(\mathrm{~d}, 8.9)$ & 137.4 & $\mathrm{C} 18, \mathrm{C} 21$ \\
\hline 17 & & 129.9 & \\
\hline 18 & & 132.1 & \\
\hline 19 & $3.32(\mathrm{~d}, 6.2)$ & 45.6 & $\mathrm{C} 7, \mathrm{C} 21, \mathrm{C} 27$ \\
\hline 20 & & 141.7 & \\
\hline 21 & & 128.1 & \\
\hline 22 & $7.80(\mathrm{~d}, 8.8)$ & 137.3 & $\mathrm{C} 20, \mathrm{C} 24$ \\
\hline 23 & $7.04(\mathrm{~d}, 8.8)$ & 115.0 & $\mathrm{C} 21, \mathrm{C} 25$ \\
\hline 24 & & 163.3 & \\
\hline 25 & & 112.4 & \\
\hline 26 & & 203.1 & \\
\hline 27 & $\begin{array}{c}2.60(\mathrm{dd}, 18.1,7.0) \\
2.65(\mathrm{dd}, 12.3,18.1)\end{array}$ & 37.8 & $\mathrm{C} 19, \mathrm{C} 25, \mathrm{C} 29$ \\
\hline 28 & $3.09 \mathrm{~m}$ & 29.7 & $\mathrm{C} 1, \mathrm{C} 26$ \\
\hline 29 & $\begin{array}{l}3.12(\mathrm{dd}, 18.7,1.8) \\
3.22(\mathrm{dd}, 6.0,18.7)\end{array}$ & 46.3 & $\mathrm{C} 2, \mathrm{C} 19, \mathrm{C} 27$ \\
\hline $3-\mathrm{OH}$ & $12.32 \mathrm{~s}$ & & $\mathrm{C} 2, \mathrm{C} 3$ \\
\hline $14-\mathrm{OH}$ & $13.99 \mathrm{~s}$ & & $\mathrm{C} 13, \mathrm{C} 14$ \\
\hline $24-\mathrm{OH}$ & $12.45 \mathrm{~s}$ & & $\mathrm{C} 24, \mathrm{C} 25$ \\
\hline
\end{tabular}


Table S2. TDDFT results for $(+)-\mathbf{1}(200<\lambda<400 \mathrm{~nm})$ after $20-\mathrm{ns}$ UV-correction. The TDDFT calculations are done at the level of B3LYP/6-31G(d,p) in the PCM model $\left(\mathrm{CH}_{3} \mathrm{CN}\right.$ solvent: dielectric constant $\varepsilon=36.64)$.

\begin{tabular}{|c|c|c|c|c|c|}
\hline Transition & $\begin{array}{c}\text { Excitation energy }^{\mathrm{a}} \\
(\mathrm{nm})\end{array}$ & $\begin{array}{l}\text { Rotatory Strength } R^{b} \\
\quad\left(10^{-40} \mathrm{cgs}\right)\end{array}$ & Oscillator Strength $f$ & $\begin{array}{c}\text { Dominant } \\
\text { Contributions }\end{array}$ & Weight \\
\hline 5 & 386.04 & 47.7365 & 0.0152 & $114 \rightarrow 121$ & 0.19 \\
\hline \multirow[t]{2}{*}{8} & 363.88 & 26.5595 & 0.0211 & $118 \rightarrow 123$ & 0.23 \\
\hline & & & & $120 \rightarrow 123$ & 0.20 \\
\hline 9 & 357.22 & -22.4980 & 0.0988 & $116 \rightarrow 121$ & 0.30 \\
\hline 13 & 333.37 & -27.4841 & 0.0617 & $113 \rightarrow 121$ & 0.23 \\
\hline 15 & 319.49 & -92.9998 & 0.2029 & $120 \rightarrow 124$ & 0.42 \\
\hline 18 & 304.89 & -29.1116 & 0.0390 & $119 \rightarrow 123$ & 0.40 \\
\hline 23 & 286.59 & 86.4117 & 0.1409 & $120 \rightarrow 126$ & 0.45 \\
\hline 30 & 266.31 & 26.8979 & 0.0459 & $117 \rightarrow 124$ & 0.31 \\
\hline 31 & 264.14 & 90.1499 & 0.0533 & $115 \rightarrow 123$ & 0.33 \\
\hline 32 & 262.16 & 44.6458 & 0.0512 & $111 \rightarrow 122$ & 0.28 \\
\hline 33 & 260.44 & 43.4817 & 0.0062 & $114 \rightarrow 123$ & 0.23 \\
\hline 34 & 258.06 & -54.1547 & 0.1629 & $114 \rightarrow 123$ & 0.23 \\
\hline 36 & 254.67 & 44.1955 & 0.0098 & $110 \rightarrow 121$ & 0.15 \\
\hline 40 & 249.59 & 48.2718 & 0.0230 & $119 \rightarrow 126$ & 0.16 \\
\hline 42 & 245.65 & -33.1234 & 0.1748 & $109 \rightarrow 121$ & 0.10 \\
\hline 46 & 242.39 & -28.0278 & 0.0812 & $111 \rightarrow 123$ & 0.27 \\
\hline 47 & 241.62 & -67.7231 & 0.1355 & $108 \rightarrow 121$ & 0.24 \\
\hline 48 & 241.13 & 57.4228 & 0.0354 & $117 \rightarrow 125$ & 0.19 \\
\hline 54 & 233.02 & 76.9117 & 0.0659 & $120 \rightarrow 129$ & 0.16 \\
\hline 56 & 231.13 & -30.4263 & 0.0163 & 118127 & 0.19 \\
\hline 58 & 229.14 & 40.7817 & 0.0534 & 107121 & 0.12 \\
\hline 59 & 227.38 & 29.0452 & 0.0212 & 119127 & 0.13 \\
\hline 65 & 222.95 & -29.7609 & 0.0377 & 115125 & 0.10 \\
\hline 71 & 216.82 & -24.5066 & 0.0067 & 118129 & 0.22 \\
\hline 74 & 214.57 & -31.8582 & 0.0459 & 112126 & 0.10 \\
\hline 75 & 214.19 & -31.5936 & 0.0621 & 103121 & 0.14 \\
\hline 84 & 207.76 & -37.2155 & 0.0705 & 108121 & 0.15 \\
\hline 87 & 205.94 & -28.5003 & 0.0524 & 117128 & 0.15 \\
\hline 89 & 204.75 & 45.5441 & 0.1363 & 117128 & 0.10 \\
\hline
\end{tabular}

${ }^{a}$ Excited states with $\mathrm{f}<0.1$ and $\mathrm{R}< \pm 20.0$ were not presented.

${ }^{\mathrm{b}}$ All the strengths were in the velocity representation.

${ }^{\mathrm{c}}$ Configurations with weights below 0.10 were not displayed. 
Table S3. cDNA synthesis method.

\begin{tabular}{cccc}
\hline Step & Reaction & Temperature & Time \\
\hline Step1 & Primer annealing & $65^{\circ} \mathrm{C}$ & $5 \mathrm{~min}$ \\
Step2 & cDNA synthesis & $42^{\circ} \mathrm{C}$ & $2 \mathrm{~h}$ \\
Step3 & Termination reaction & $70^{\circ} \mathrm{C}$ & $15 \mathrm{~min}$ \\
\hline
\end{tabular}

Table S4. Primers for PCR.

\begin{tabular}{|c|c|c|}
\hline & Forward primer & Reverse prime \\
\hline Nestin & AAGGGGATGCCGGGAAGGAAGACTG & CAGAGGCCCCTGACCCCCACCACT \\
\hline NSE & GCCCGTGCCGGCCTTTAATGTGATC & ATCGGGAAGGGTCAGCGGGAGACTT \\
\hline
\end{tabular}




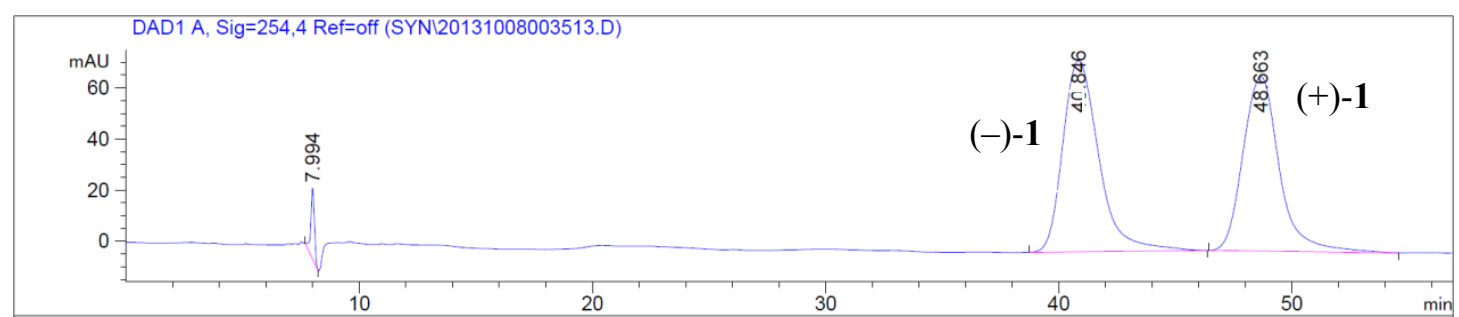

Figure S1. Chiral HPLC separation of selesconol (1).

$<$ Column Performance Report $>$

Column: CHIRALPAK ${ }^{\circledR}$ IA $^{\text {TM }}, 0.50 \mathrm{~cm} \mathrm{ID.} \times 25 \mathrm{~cm} \mathrm{~L}, 5 \mu \mathrm{m}$

Mobile Phase: $\mathrm{Hex} / \mathrm{EtOH}=50 / 50(\mathrm{v} / \mathrm{v})$

$\begin{array}{cccc}\text { Peak No. } & \text { Time } & \text { Area } & \text { Area \% } \\ 1 & 40.846 & 8035 & 51.0852 \\ 2 & 48.663 & 7394 & 47.0114\end{array}$
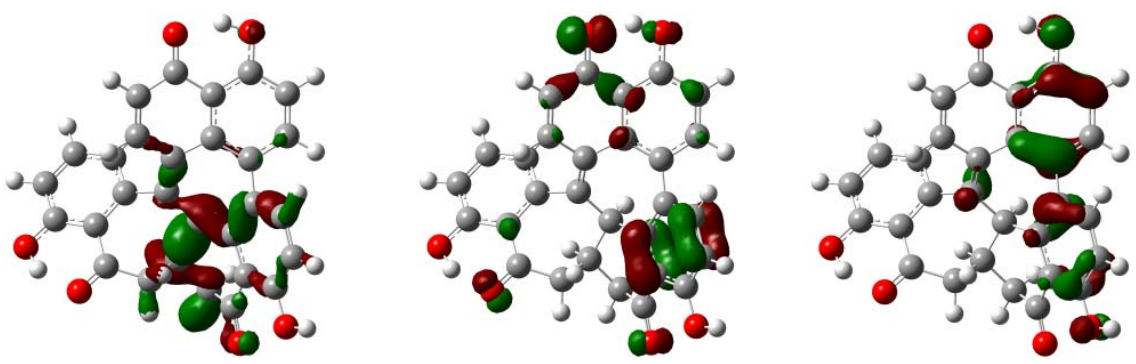

MO $109(-8.98 \mathrm{eV})$

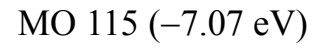

MO $120(-5.66 \mathrm{eV})$
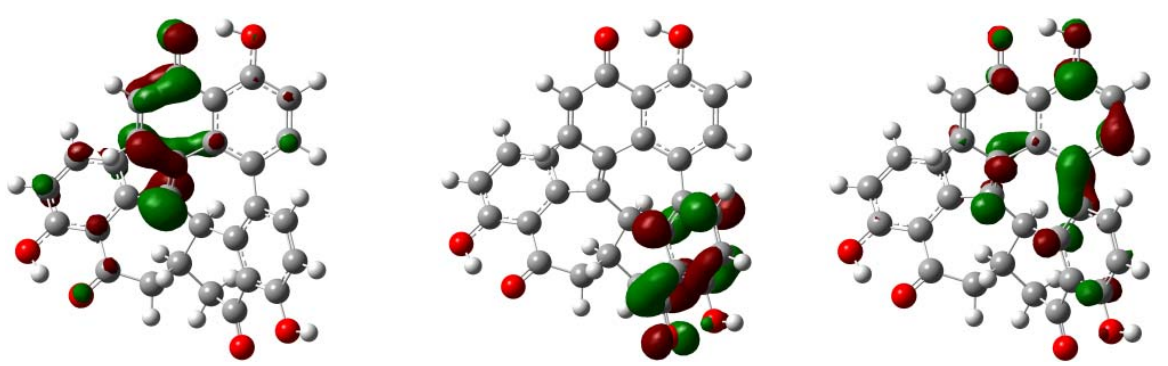

MO $121(-2.69 \mathrm{eV}) \quad$ MO $123(-1.55 \mathrm{eV}) \quad$ MO $124(-0.98 \mathrm{eV})$
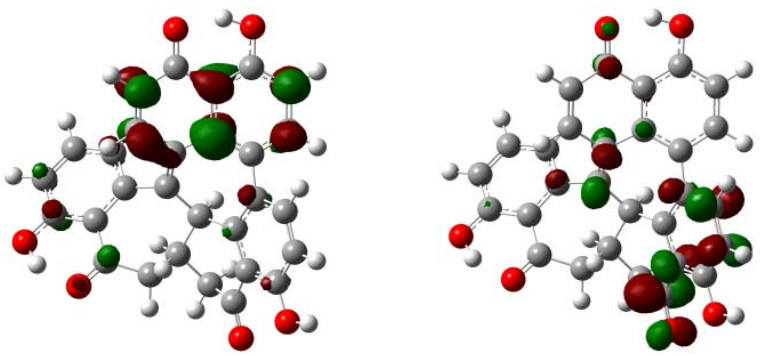

MO $126(-0.46 \mathrm{eV}) \quad$ MO $129(0.79 \mathrm{eV})$

Figure S2. The most important orbitals of $(+)$-1. The optimized conformation is obtained at B3LYP/6-31G(d,p) level in the PCM $\left(\mathrm{CH}_{3} \mathrm{CN}\right.$ solvent: dielectric constant $\left.\varepsilon=36.64\right)$ model. 


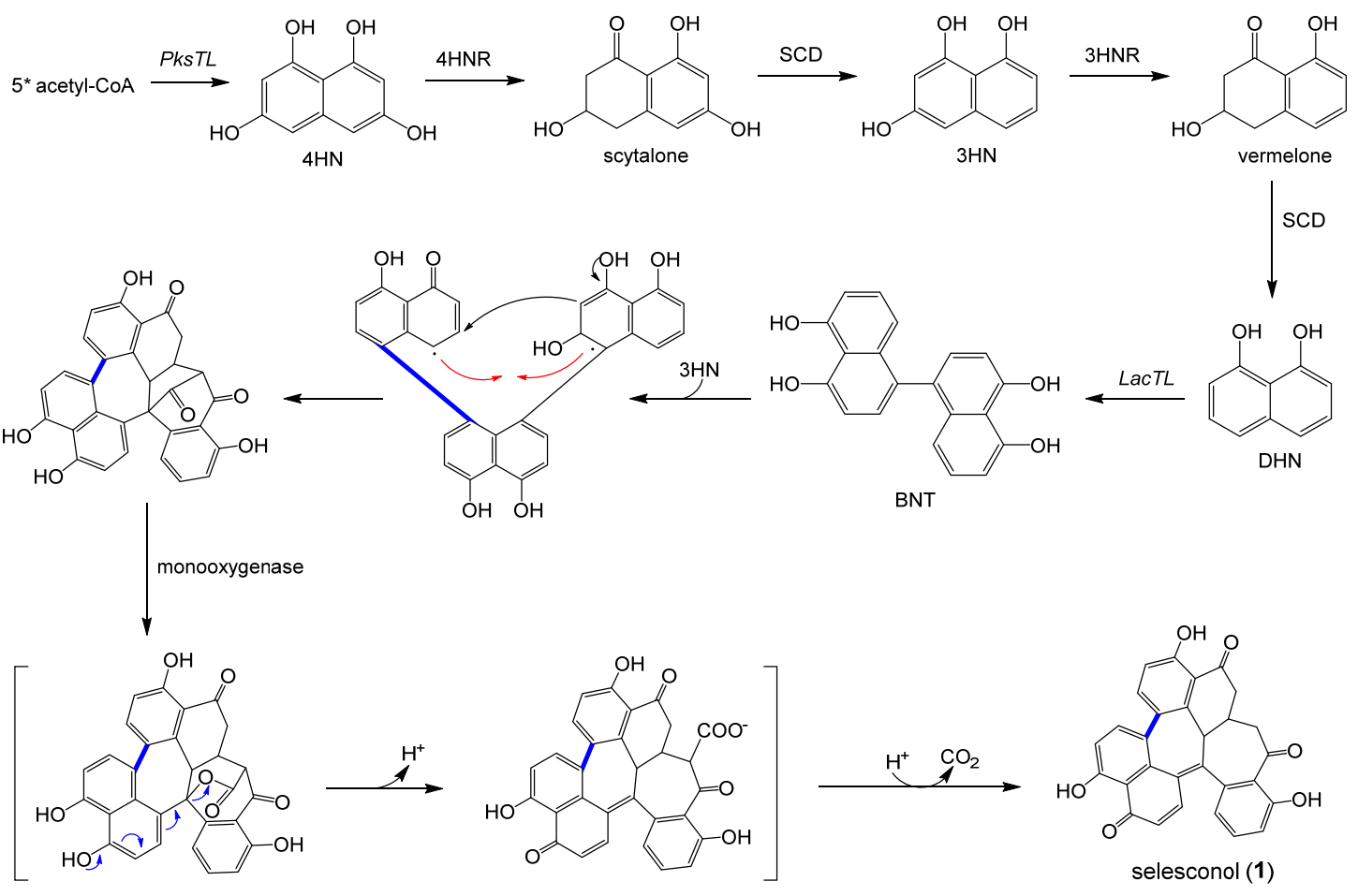

Figure S3. Metabolic pathway towards selesconol (1). The biosynthetic steps including polyketide synthase (pksTL)- and laccase (lacTL)-catalyzed generation and coupling of 1,3,8-trihydroxy- (3HN), 1,8-dihydroxy-naphthalenes (DHN) and 1,1'-binaphthalene-4,4',5,5'-tetrol (BNT). 4HN, 1,3,6,8-tetrahydroxy-naphthalene; $4 \mathrm{HNR}, 4 \mathrm{HN}$ reductase; $3 \mathrm{HNR}$, $3 \mathrm{HN}$ reductase; and $\mathrm{SCD}$, scytalone dehydratase. 3

a)

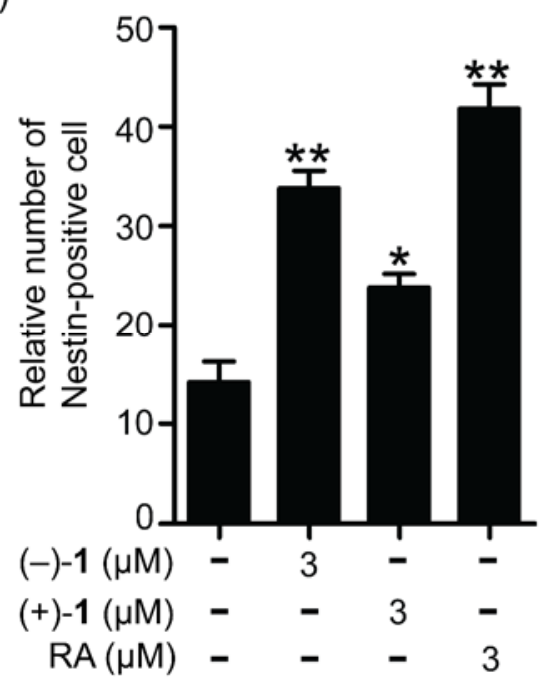

b)

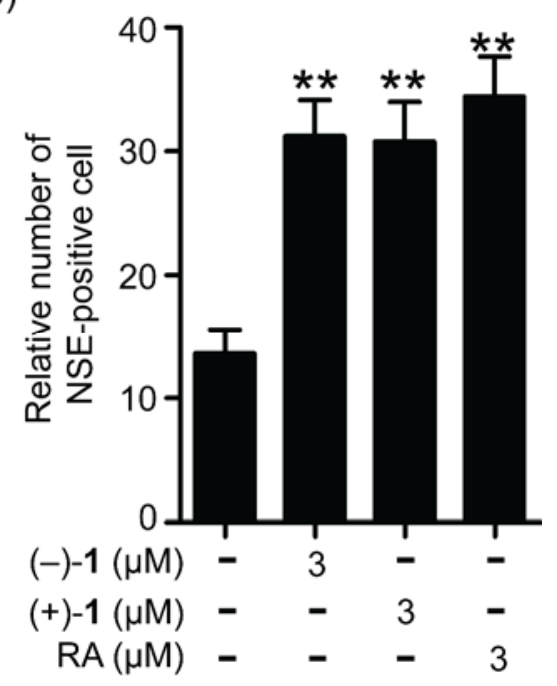

Figure S4. Immunofluorescence analysis of Nestin-positive and NSE-positive cells treated separately with (+)-1, (-)-1 and RA. The measurement was accomplished by counting five random fields per group. 


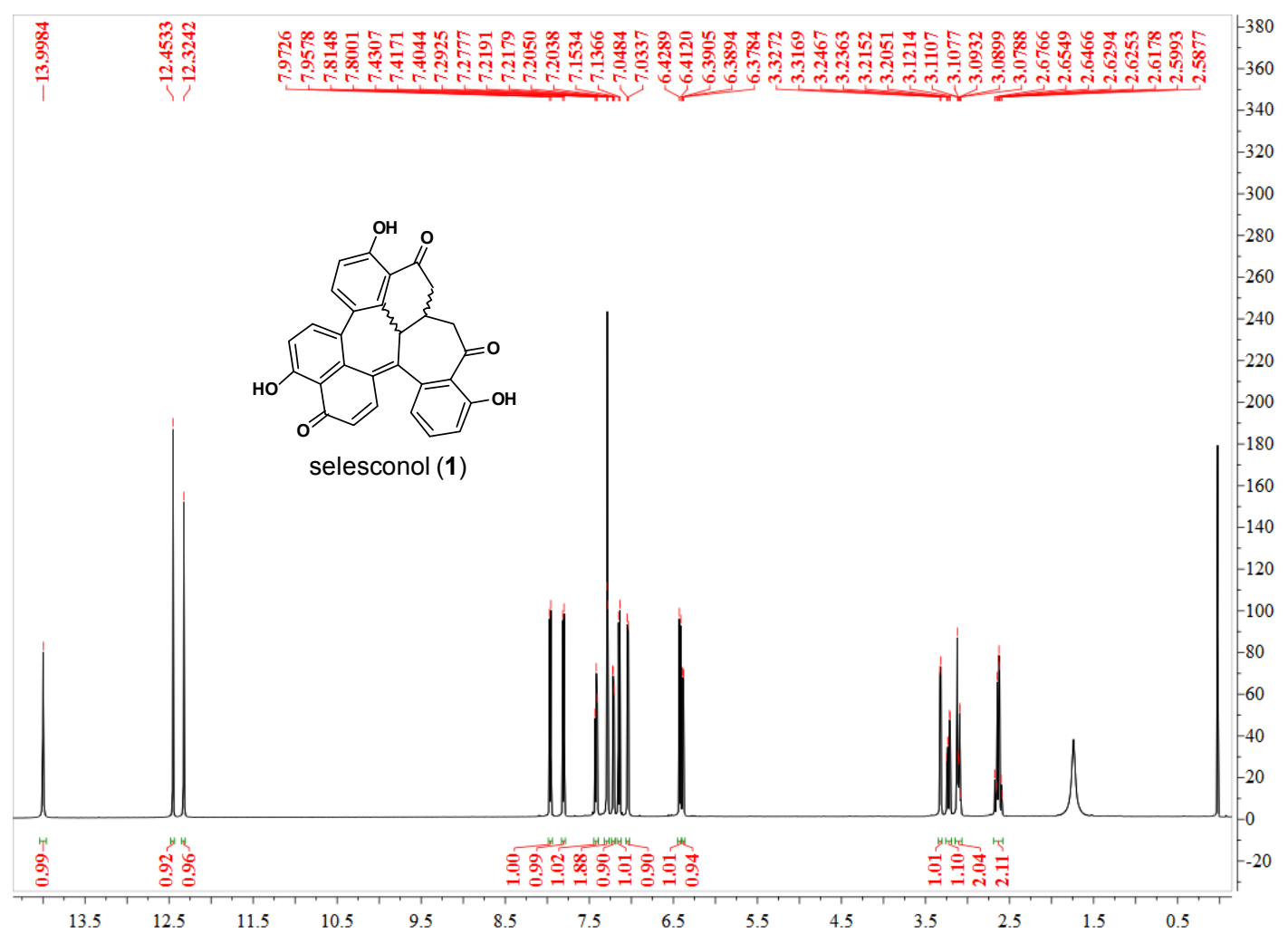

Figure S5. ${ }^{1} \mathrm{H}-\mathrm{NMR}$ spectrum of selesconol $\left(\mathbf{1}, 600 \mathrm{MHz}, \mathrm{CDCl}_{3}\right)$.

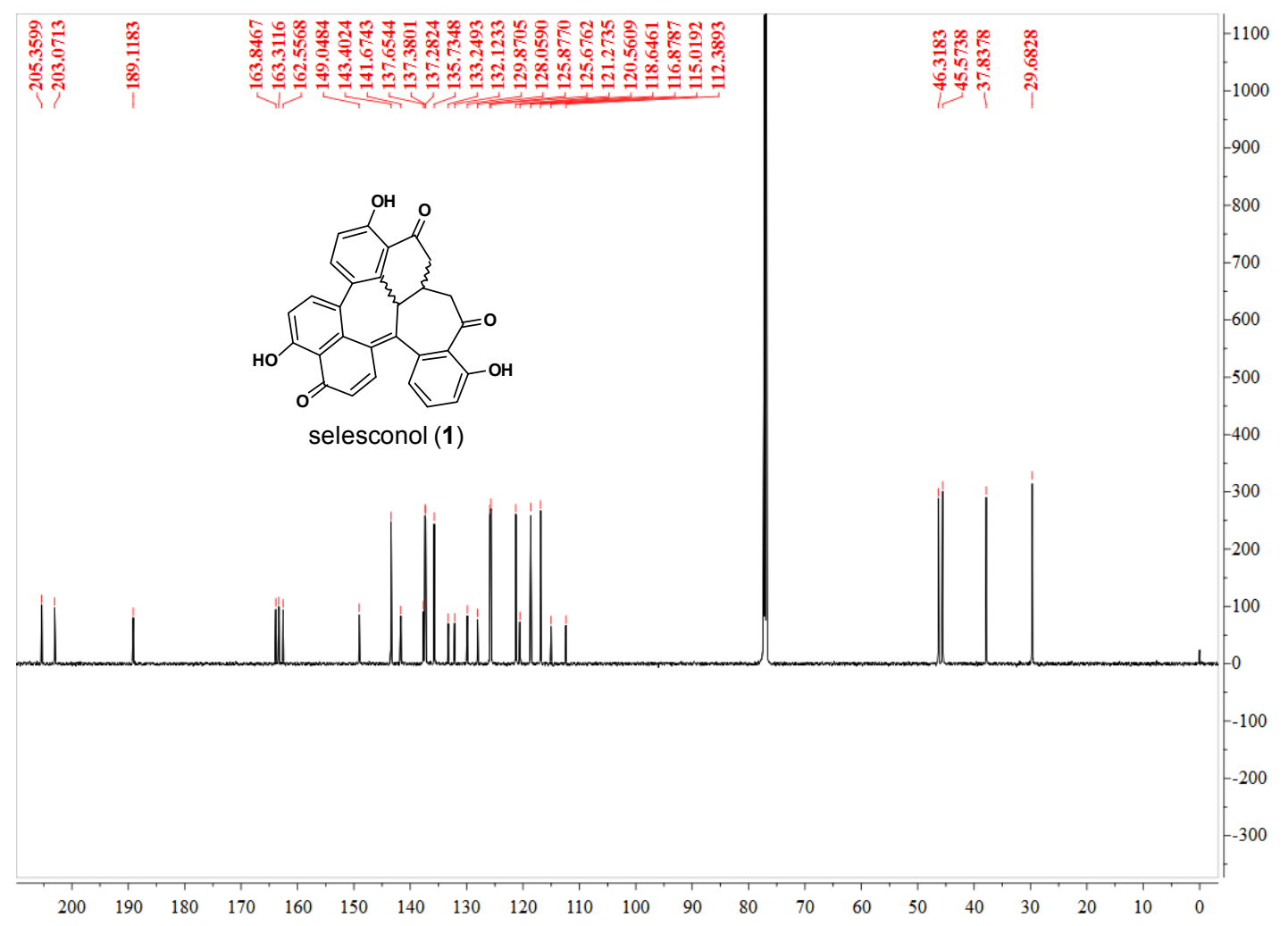

Figure S6. ${ }^{13} \mathrm{C}$-NMR spectrum of selesconol $\left(\mathbf{1}, 150 \mathrm{MHz}, \mathrm{CDCl}_{3}\right)$. 


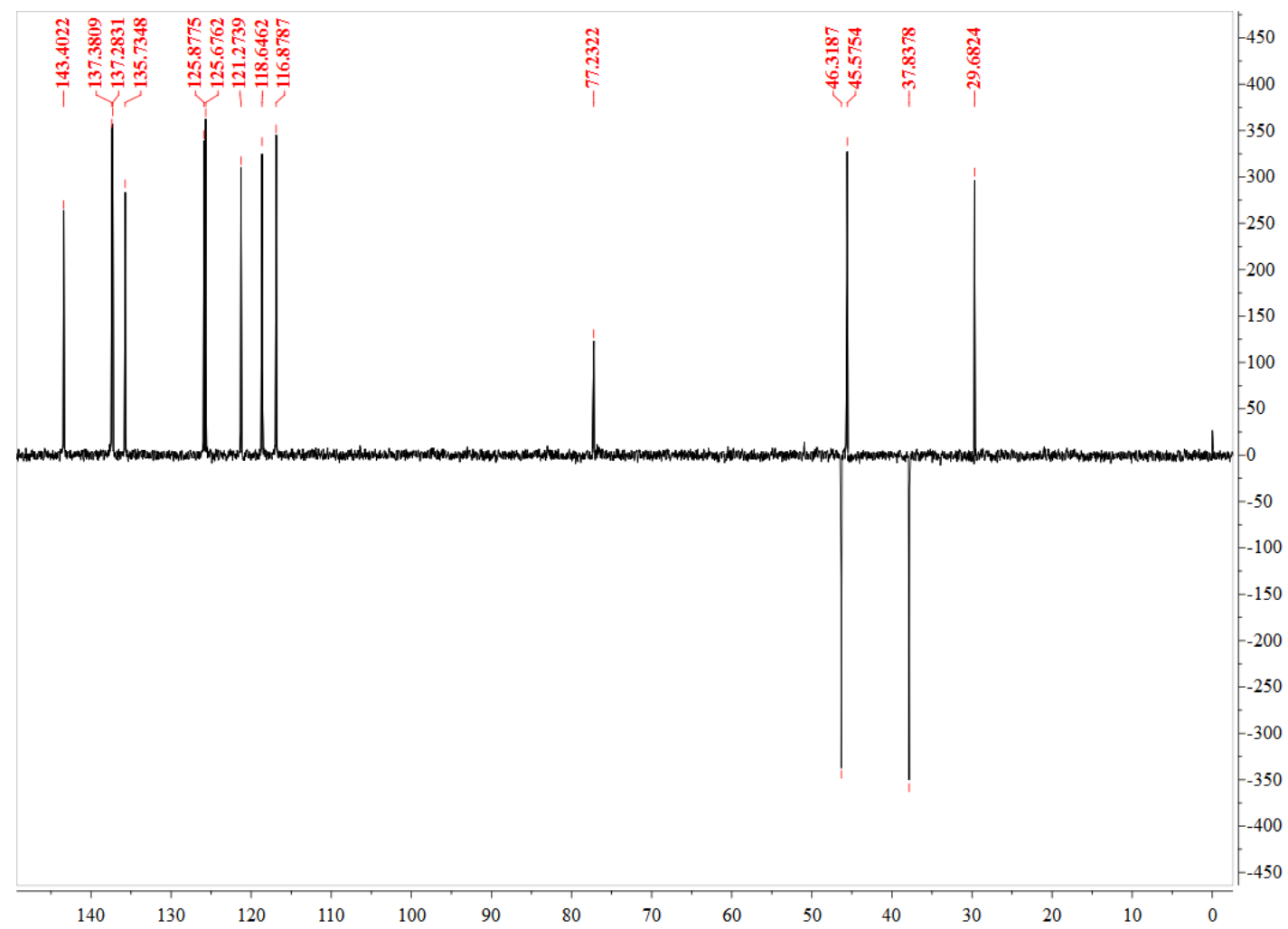

Figure S7. DEPT spectrum of selesconol $\left(1, \mathrm{CDCl}_{3}\right)$.

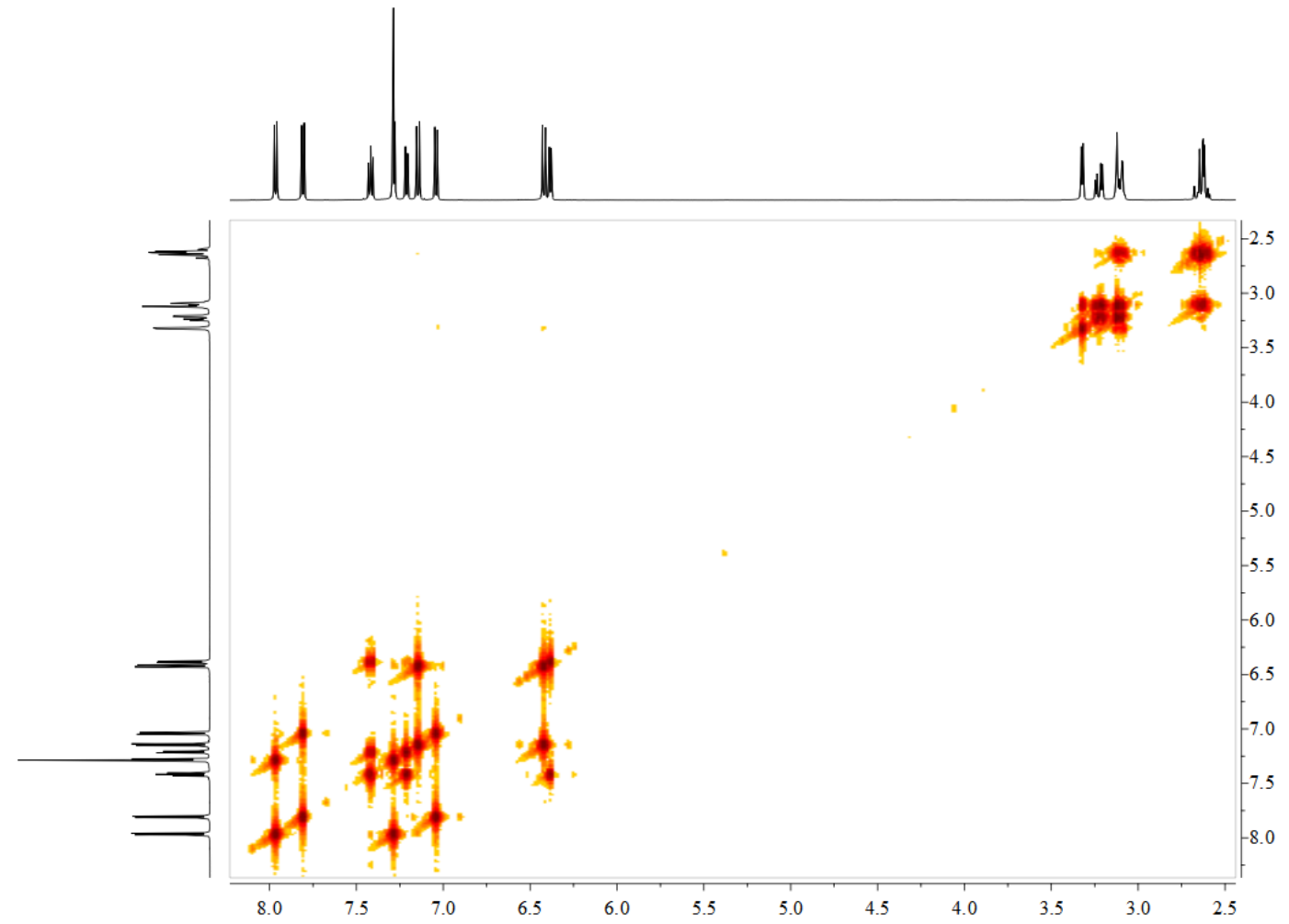

Figure S8. ${ }^{1} \mathrm{H}-{ }^{1} \mathrm{H} C O S Y$ spectrum of selesconol $\left(\mathbf{1}, \mathrm{CDCl}_{3}\right)$. 


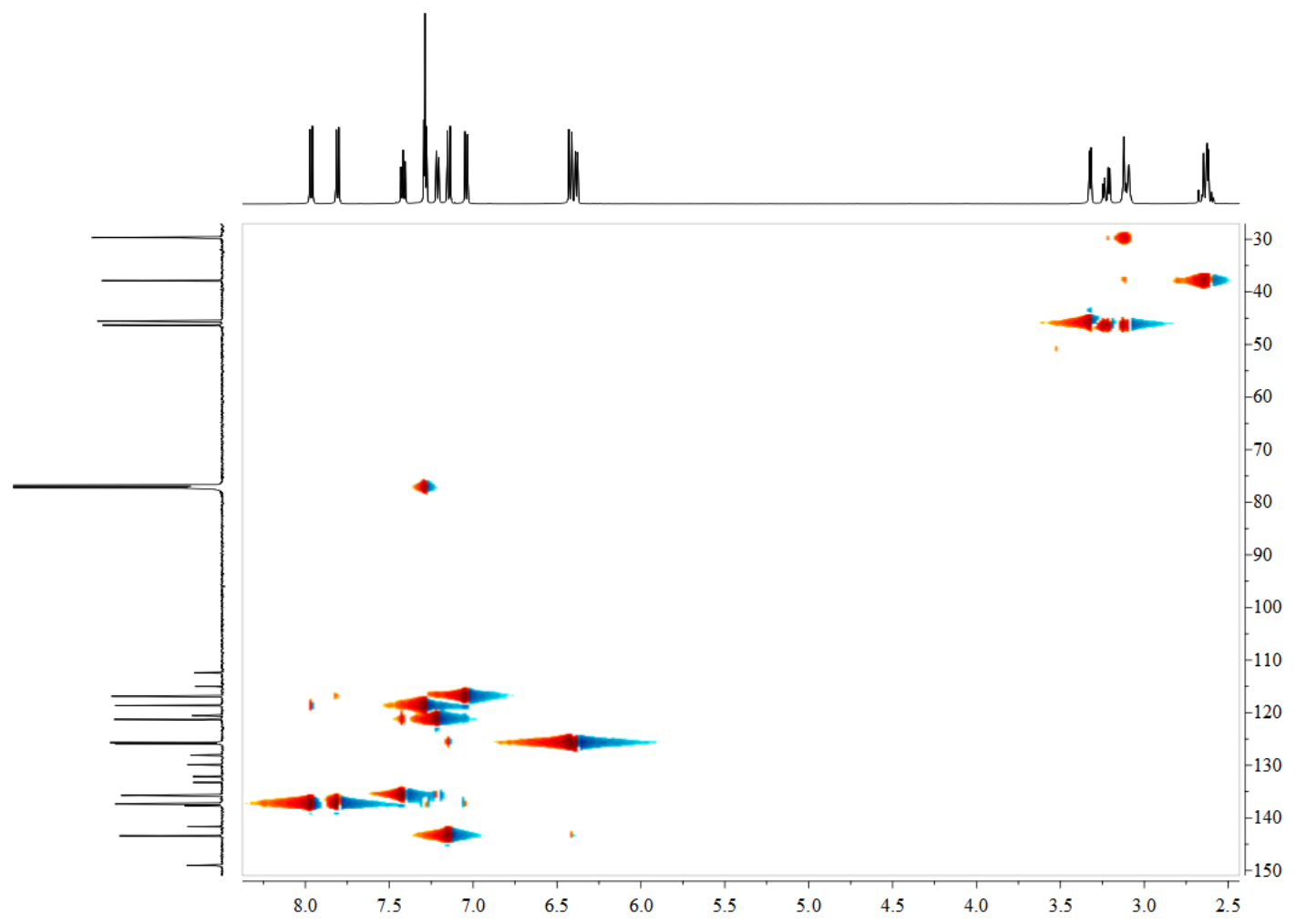

Figure S9. HSQC spectrum of selesconol $\left(\mathbf{1}, \mathrm{CDCl}_{3}\right)$.

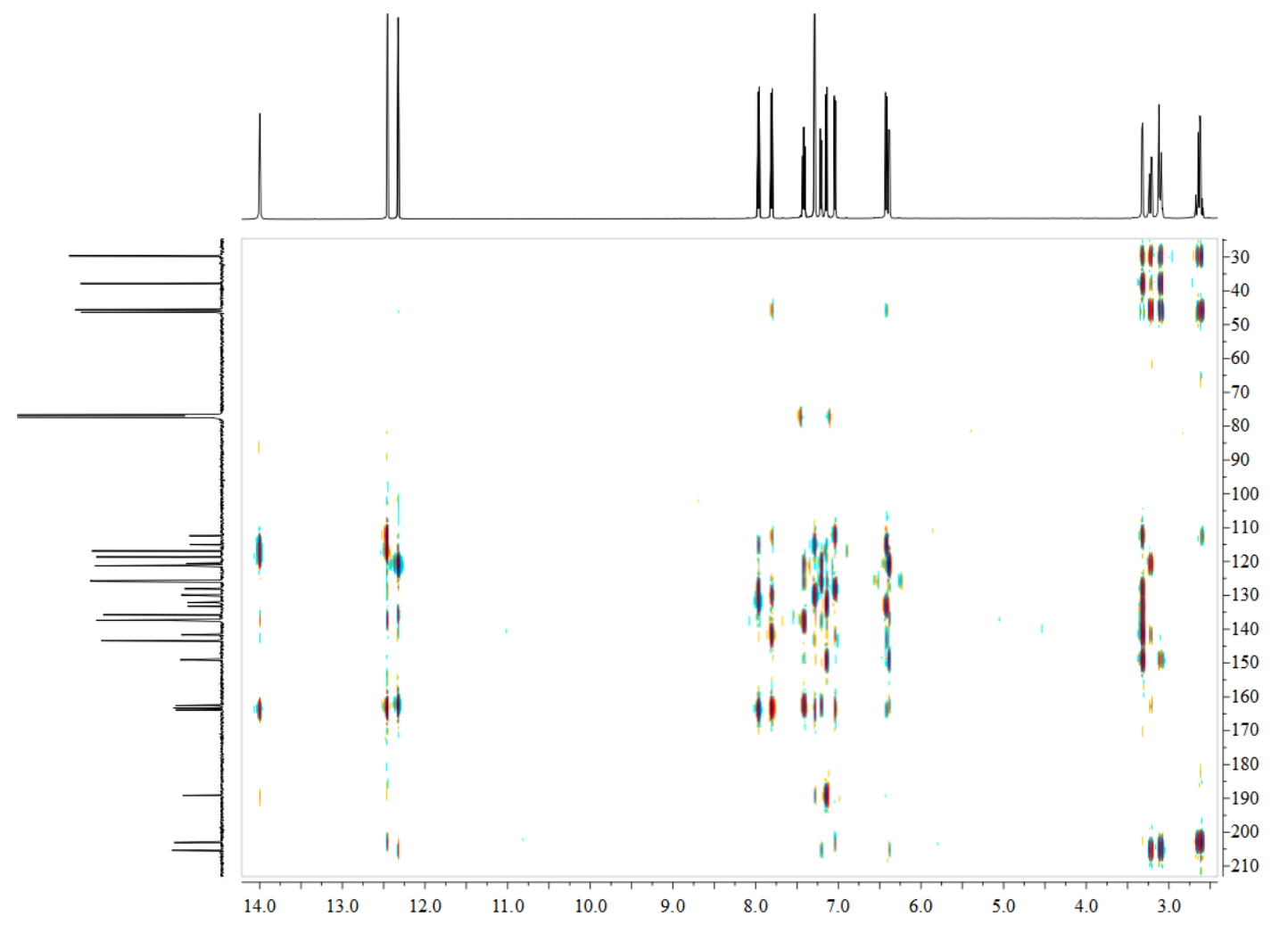

Figure S10. HMBC spectrum of selesconol $\left(1, \mathrm{CDCl}_{3}\right)$. 


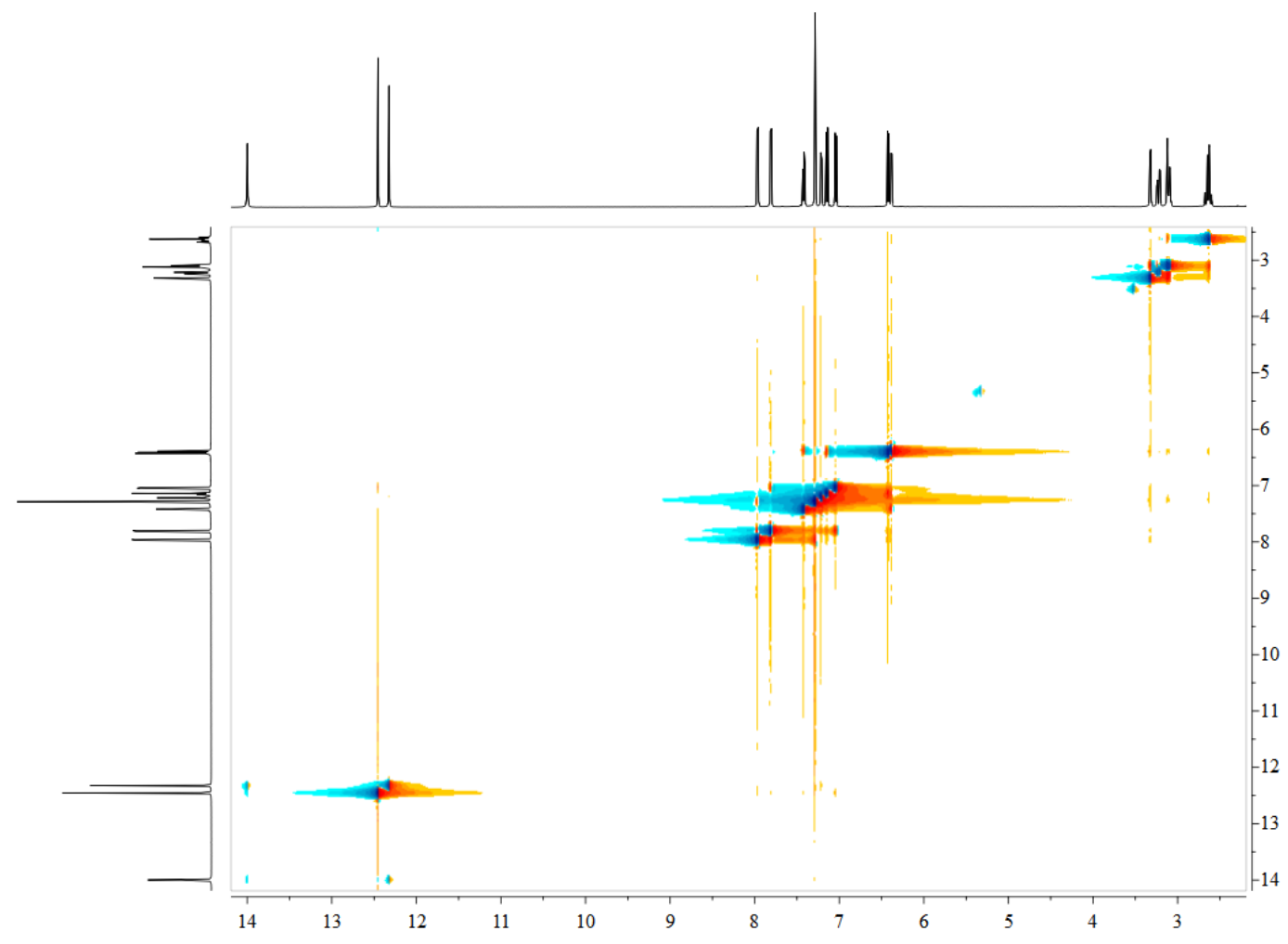

Figure S11. NOSEY spectrum of selesconol $\left(1, \mathrm{CDCl}_{3}\right)$. 